K. Nomizu, U. Pinkall and F. Podestà

Nagoya Math. J.

Vol. 120 (1990), 205-222

\title{
ON THE GEOMETRY OF AFFINE KÄHLER IMMERSIONS
}

\author{
KATSUMI NOMIZU, ULRICH PINKALL AND FABIO PODESTÀ
}

In this paper we extend the work on affine immersions [N-Pi]-1 to the case of affine immersions between complex manifolds and lay the foundation for the geometry of affine Kähler immersions. The notion of affine Kähler immersion extends that of a holomorphic and isometric immersion between Kähler manifolds and can be contrasted to the notion of holomorphic affine immersion which has been established in the work of Dillen, Vrancken and Verstraelen [D-V-V] and that of Abe [A].

In Section 1 we discuss the groundwork for complex affine immersions and in Sections 2 and 3 for affine Kähler immersions. We shall give two parallel formulations, one in terms of real vector fields and the other in terms of vector fields of type $(1,0)$. The former is useful in establishing the fundamental theorem for affine Kähler immersions as we do in Section 4. The latter is useful in getting examples of complex manifolds with affine Kähler connections, as was done in [N-Po], and in obtaining, in Sections 5 and 6, several theorems on affine Kähler immersions into the complex Euclidean space, including an analogue of the classical theorem of Pick and Berwald on vanishing cubic forms.

\section{§1. Complex connections and affine immersions}

Let $M$ be an $n$-dimensional complex manifold with complex structure $J$. We consider a torsion-free linear connection $\nabla$ which is compatible with $J$, that is, $\nabla J=0$. Such a connection as covariant differentiation

$$
(X, Y) \in \mathbf{X} \times \mathbf{X} \mapsto \nabla_{X} Y \in \mathbf{X}
$$

with usual properties can be extended to a complex linear connection with covariant differentiation

$$
(X, Y) \in \mathbf{X}^{c} \times \mathbf{X}^{c} \mapsto \nabla_{X} Y \in \mathbf{X}^{c},
$$

where $\mathrm{X}^{c}$ is the space of all complex vector fields $X+i Y$ with $X, Y \in X$.

Received November 25, 1989. 
We have then

$$
\nabla_{Z} \bar{W}=\overline{\nabla_{Z} W} \text {. }
$$

We write $\mathbf{X}^{(1,0)}$ (resp. $\mathbf{X}^{(0,1)}$ ) for the set of all vector fields of type $(1,0)$ (resp. $(0,1)$ ). Since $\nabla$ is compatible with $J$, it follows that $\nabla_{z}$, where $Z \in \mathbf{X}$, maps each of $\mathbf{X}^{(1,0)}$ and $\mathbf{X}^{(0,1)}$ into itself. Conversely, it can be checked that a torsion-free complex linear connection with these properties comes from a torsion-free linear connection compatible with $J$. In the following, we shall always consider this kind of linear connection $\nabla$, expressed in terms of real vector fields or complex vector fields.

We shall say that $\nabla$ is affine Kähler if its curvature tensor has the property

$$
R(J X, J Y)=R(X, Y) \quad \text { for all } X, Y \in \mathbf{X} .
$$

This condition is equivalent to

$$
R(Z, W)=0 \quad \text { for all } Z, W \in \mathbf{X}^{(1,0)} .
$$

Affine Kähler connections are to contrasted with holomorphic connections whose curvature tensors satisfy

$$
R(J X, Y)=J R(X, Y) \quad \text { for all } X, Y \in \mathbf{X}
$$

or equivalently

$$
R(Z, \bar{W})=0 \quad \text { for all } Z, W \in \mathbf{X}^{(1,0)} .
$$

We now consider an $(n+1)$-dimensional complex manifold $\tilde{M}$ with a torsion-free affine connection $\tilde{\nabla}$ compatible with the complex structure $J$. Let $f: M \rightarrow \tilde{M}$ be a holomorphic immersion; we wish to consider how we may induce an affine connection $\nabla$ on $M$ with zero torsion and compatible with the complex structure on $M$, which we shall denote by the same symbol $J$.

First in terms of real vector fields, we choose a real transversal vector field $\xi$ to $M$. Then $J \xi$ is also transversal. Hence for vector fields $X$ and $Y$ on $M$ we may write

$$
\tilde{\nabla}_{X}\left(f_{*} Y\right)=f_{*}\left(\nabla_{X} Y\right)+h(X, Y) \xi+k(X, Y) J \xi,
$$

thus defining a torsion-free affine connection $\nabla$ and covariant symmetric tensors $h$ and $k$ on $M$. Since $f$ is holomorphic, we have

$$
f_{*}(J Y)=J\left(f_{*} Y\right)
$$


therefore from $\tilde{\nabla} J=0$ we obtain $\nabla J=0$ as well as

$$
k(X, Y)=-h(J X, Y) .
$$

Since $k$ and $h$ are both symmetric, we have

$$
h(J X, Y)=h(X, J Y), \quad k(J X, Y)=k(X, J Y) .
$$

We shall also write

$$
\tilde{\nabla}_{X} \xi=-f_{*}(A X)+\mu(X) \xi+\nu(X) J \xi,
$$

thus defining a $(1,1)$-tensor $A$ and two 1 -forms $\mu$ and $\nu$ on $M$. We get

$$
\nabla_{X}(J \xi)=-J A(X)-\nu(X) \xi+\mu(X) J \xi .
$$

We shall now find formulas in terms of complex vector fields. For $X, Y$ $\epsilon \mathrm{X}$, we write $Z=X-i J X$ and $W=Y-i J Y$. We expand and simplify

$$
\tilde{\nabla}_{Z}\left(f_{*} W\right)=\tilde{\nabla}_{X-i J X}\left(f_{*}(Y-i J Y)\right)
$$

by using (1.1)-(1.4). The tangential component of (1.7) is equal to

$$
\nabla_{Z} W=\nabla_{X-i J X}(Y-i J Y) \text {. }
$$

The transversal component of (1.7) can be expressed in the form

$$
2[h(X, Y)+i k(X, Y)](\xi-i J \xi) .
$$

We introduce a transversal $(1,0)$-vector field

$$
\zeta=\xi-i J \xi \text {. }
$$

By extending $h$ as a complex bilinear function on complex tangent vectors we get

$$
h(Z, W)=2(h(X, Y)+i k(X, Y)) .
$$

Using (1.10) and (1.11) we may rewrite the transversal component (1.9) in the following form

$$
h(Z, W) \zeta
$$

From (1.7), (1.8) and (1.9') we get

$$
\tilde{\nabla}_{z}\left(f_{*}(W)\right)=f_{*}\left(\nabla_{z} W\right)+h(Z, W) \zeta .
$$

Now we compute $\tilde{\nabla}_{z}\left(f_{*}(\bar{W})\right)$ and find

$$
\tilde{\nabla}_{z}\left(f_{*}(\bar{W})\right)=f_{*}\left(\nabla_{z} \bar{W}\right)
$$


without the transversal component on the right-hand side. This corresponds to

$$
h(Z, \bar{W})=0,
$$

which can be directly verified for the complex extension $h$.

We also note that, for any complex vectors $Z$ and $W$ in general, $h(\bar{Z}, \bar{W})$ is conjugate to $h(Z, W), \nabla_{Z} \bar{W}$ is conjugate to $\nabla_{Z} W$, and we have the formulas conjugate to (1.12), (1.13) and (1.14).

Now we work with the complex versions of (1.5) and (1.6). By computation we get

$$
\tilde{\nabla}_{z} \zeta=-(A-i J A)(Z)+(\mu+i \nu)(Z) \zeta
$$

so that

$$
\tilde{\nabla}_{z} \zeta=-S(Z)+\tau(Z) \zeta
$$

where

$$
S=A-i J A, \quad \tau=\mu+i \nu .
$$

On the other hand we have

$$
\begin{aligned}
& \tilde{\nabla}_{\bar{Z}} \zeta=-S(\bar{Z})+\tau(\bar{Z}) \zeta \\
& \tilde{\nabla}_{z} \bar{\zeta}=-\bar{S}(Z)+\tau(Z) \bar{\zeta} \\
& \tilde{\nabla}_{\bar{Z}} \bar{\zeta}=-\bar{S}(\bar{Z})+\tau(\bar{Z}) \bar{\zeta},
\end{aligned}
$$

where by definition

$$
\bar{S}=A+i J A, \quad \bar{\tau}=\mu-i \nu .
$$

We easily see that the conjugate of $S(Z)$ is $\bar{S}(\bar{Z})$ and the conjugate of $\tau(Z)$ is $\bar{\tau}(\bar{Z})$.

\section{§2. Antiholomorphic transversal vector field and affine Kähler immersions}

We recall here that in $\mathbf{C}^{n}$ a vector field $Z=\sum_{k=1}^{n} \phi^{k} \partial / \partial z^{k}$ in $\mathbf{X}^{(1,0)}$ is said to be holomorphic or antiholomorphic if each function $\phi^{k}$ is holomorphic or antiholomorphic. The notion of holomorphic vector field can, of course, be extended to the case of an arbitrary complex manifold, but that is not the case with antiholomorphic vector fields. We make the following definition. 
Let $M$ be a complex manifold with a torsionfree affine connection $\nabla$ compatible with the complex structure $J$. We shall say that a vector field $\zeta$ of type $(1,0)$ is antiholomorphic if $\nabla_{z} \zeta=0$ for every tangent vector of type $(1,0)$. If $\zeta$ is antiholomorphic and if $\phi$ is an antiholomorphic function, then $\phi \zeta$ is antiholomorphic. The set of antiholomorphic vector fields of type $(1,0)$ forms a vector space over $\mathbf{C}$ and for $\mathbf{C}^{n}$ this notion coincides with the obvious one.

Let $\zeta=\xi-i J \xi$ and $Z=X-i J X$, where $\xi$ and $X$ are real vector fields; then $\nabla_{Z} \zeta=\nabla_{X} \xi-\nabla_{J X} J \xi-i \nabla_{J X} \xi-i \nabla_{X} J \xi$ is zero if and only if $\nabla_{J X} \xi=-J \nabla_{X} \xi$. Therefore we have the following characterization:

$$
\zeta \text { is antiholomorphic if and only if } \nabla_{I X} \xi=-J \nabla_{X} \xi \text { for every } X \text {. }
$$

This can be compared to the well-known fact that $\zeta$ is holomorphic if and only if $[\xi, J X]=J[\xi, X]$ for every $X$, which can be rephrased: if and only if $\nabla_{J X} \xi=J \nabla_{X} \xi$ for every $X$.

For the complex immersion $f: M \rightarrow \tilde{M}$ a $(1,0)$ vector field $\zeta$ along $f$ is said to be antiholomorphic if $\tilde{\nabla}_{Z} \zeta=0$ for every $(1,0)$-vector field $Z$ on $M$.

Remark. All the work so far on affine differential geometry of complex hypersurfaces depends on the choice of a holomorphic transversal vector field (see [DFV]). We know, however, that this premise does not hold for a kählerian hypersurface; namely if $f: M \rightarrow \tilde{M}$ is a holomorphic immersion of a kählerian manifold $M$ into $\tilde{M}$, then the choice of a holomorphic $(1,0)$-tranversal vector field $\zeta$ leads to a holomorphic connection on $M$, which cannot agree with the connection from the induced kählerian metric unless it is flat. On the other hand, for any kählerian hypersurface we can choose an antiholomorphic $(1,0)$-transversal vector field.

Suppose now we have chosen an antiholomorphic $(1,0)$-transversal vector field $\zeta$ along $f$. With such a choice we call $f$ an affine Kähler immersion. We have that

$$
\tilde{\nabla}_{J X} \xi=-f_{*}(A J X)+\mu(J X) \xi+\nu(J X) J \xi,
$$

but by (2.1) this is equal to

$$
-J \tilde{\nabla}_{X} \xi=f_{*}(J A X)+\nu(X) \xi-\mu(X) J \xi
$$

so that for every $X$ we have

$$
A J=-J A
$$




$$
\nu(X)=\mu(J X) .
$$

Actually, (2.2) and (2.3) are necessary and sufficient for $\zeta$ to be antiholomorphic.

\section{§3. Curvature tensors on the hypersurface in $\mathbf{C}^{n+1}$}

From now on we shall assume that we take a $(1,0)$-antiholomorphic transversal vector field for our immersion $f$. We derive basic equations for geometry of the complex hypersurfaces in $\mathbf{C}^{n+1}$. We summarize the basic formulas (omitting $f_{*}$ where there is no danger of confusion and also writing $D$ for covariant differentiation in $\left.\mathbf{C}^{n+1}\right) ; X, Y, Z, W$ will be (1, 0)-vector fields on $M$ :

(Ia) $D_{Z} W=\nabla_{Z} W+h(Z, W) \zeta$ and its conjugate,

(Ib) $D_{z} \bar{W}=\nabla_{Z} \bar{W}$ and its conjugate.

Since $h$ is defined or all complex vectors, it is worth noting that

(Ic) $h(Z, \bar{W})=h(\bar{Z}, W)=0$,

(IIa) $D_{Z} \zeta=0$ and its conjugate,

(IIb) $D_{\bar{Z}} \zeta=-S(\bar{Z})+\tau(\bar{Z}) \zeta$ and its conjugate.

It is useful to note that

(IIc) $S(Z)=\bar{S}(\bar{Z})=0, \quad \tau(Z)=\bar{\tau}(\bar{Z})=0$.

To be sure, the conjugate of (IIb) reads:

$$
D_{z} \bar{\zeta}=-\bar{S}(Z)+\bar{\tau}(Z) \bar{\zeta},
$$

where $\bar{S}$ and $S$ are related by

$$
\bar{S}(Z)=\overline{S(\bar{Z})},
$$

and $\tau$ and $\tau$ are related by

$$
\bar{\tau}(Z)=\overline{\tau(\bar{Z})}
$$

We now compute the curvature tensor of $\nabla$. Using (Ia) and (IIa) we have

$$
\begin{aligned}
D_{X} D_{Y} Z & =D_{X}\left(\nabla_{Y} Z+h(Y, Z) \zeta\right) \\
& =\nabla_{X} \nabla_{Y} Z+h\left(X, \nabla_{Y} Z\right) \zeta+X(h(Y, Z)) \zeta+h(Y, Z) D_{X} \zeta \\
& =\nabla_{X} \nabla_{Y} Z+h\left(X, \nabla_{Y} Z\right) \zeta+\left(\nabla_{X} h\right)(Y, Z) \zeta+h\left(\nabla_{X} Y, Z\right) \zeta+h\left(Y, \nabla_{X} Z\right) \zeta
\end{aligned}
$$

and a similar equation where $X$ and $Y$ are interchanged. Also

$$
D_{[X, Y]} Z=\nabla_{[X, Y]} Z+h[(X, Y], Z) \zeta .
$$


From these equations we obtain

$$
0=R(X, Y) Z+\left[\left(\nabla_{X} h\right)(Y, Z)-\left(\nabla_{Y} h\right)(X, Z)\right] \zeta .
$$

Therefore

$$
\begin{gathered}
R(X, Y) Z=0 \\
\left(\nabla_{X} h\right)(Y, Z)=\left(\nabla_{Y} h\right)(X, Z) .
\end{gathered}
$$

We have also the conjugates of these equations. Now we have, by using (Ib) and (IIb)

$$
\begin{aligned}
& D_{X} D_{\bar{Y}} Z=D_{X}\left(\nabla_{\bar{Y}} Z\right)=\nabla_{X} \nabla_{\bar{Y}} Z+h\left(X, \nabla_{Y} Z\right), \\
D_{\bar{Y}} D_{X} Z= & D_{\bar{Y}}\left(\nabla_{X} Z+h(X, Z) \zeta\right) \\
= & \nabla_{\bar{Y}} \nabla_{X} Z+\left\{\left(\nabla_{\bar{Y}} h\right)(X, Z)+h\left(\nabla_{\bar{Y}} X, Z\right)+h\left(X, \nabla_{\bar{Y}} Z\right)\right\} \zeta+ \\
& -h(X, Z) S(\bar{Y})+\tau(\bar{Y}) \zeta,
\end{aligned}
$$

and

$$
D_{[X, \bar{Y}]} Z=\nabla_{[X, \bar{Y}]} Z+h([X, \bar{Y}], Z) \zeta .
$$

From these equations we obtain

$$
\begin{aligned}
0= & R(X, \bar{Y}) Z+h(X, Z) S(\bar{Y})+ \\
& -\left\{\left(\nabla_{\bar{Y}} h\right)(X, Z)+h\left(\nabla_{\bar{Y}} X, Z\right)+h([X, \bar{Y}], Z)-h(X, Z) \tau(\bar{Y})\right\} \zeta,
\end{aligned}
$$

where

$$
h\left(\nabla_{\bar{Y}} X, Z\right)+h([X, \bar{Y}], Z)=h\left(\nabla_{X} \bar{Y}, Z\right)=0
$$

by (Ic), because $\nabla_{X} \bar{Y}$ is of type $(0,1)$. Thus we get

$$
\begin{gathered}
R(X, \bar{Y}) Z=-h(X, Z) S(\bar{Y}), \\
\left(\nabla_{\bar{Y}} h\right)(X, Z)+\tau(\bar{Y}) h(X, Z)=0 .
\end{gathered}
$$

The conjugate equations read:

$$
\begin{gathered}
R(X, \bar{Y}) \bar{Z}=h(\bar{Y}, \bar{Z}) \bar{S}(X), \\
\left(\nabla_{X} h\right)(\bar{Y}, \bar{Z})+\bar{\tau}(X) h(\bar{Y}, Z)=0 .
\end{gathered}
$$

Next we have

$$
D_{X} D_{Y} \bar{Z}=D_{X}\left(\nabla_{Y} \bar{Z}\right)=\nabla_{X} \nabla_{Y} \bar{Z}+h\left(X, \nabla_{Y} \bar{Z}\right) \zeta=\nabla_{X} \nabla_{Y} \bar{Z},
$$

since $h\left(X, \nabla_{X} \bar{Z}\right)=0$ by (Ic). Moreover 


$$
\begin{gathered}
D_{Y} D_{X} \bar{Z}=D_{Y}\left(\nabla_{X} \bar{Z}\right)=\nabla_{Y} \nabla_{X} \bar{Z} \\
D_{[X, Y]} \bar{Z}=\nabla_{[X, Y]} \bar{Z}
\end{gathered}
$$

again using the fact that $[X, Y] \in \mathbf{X}^{(1,0)}$ and $\bar{Z} \in \mathbf{X}^{(0,1)}$. From these equations we get

$$
R(X, Y) \bar{Z}=0
$$

The conjugate equation reads

$$
R(\bar{X}, \bar{Y}) Z=0 .
$$

For our complex hypersurface we summarize

Equations of Gauss and Codazzi

(IIIa) $R(X, Y)=0$ for $X, Y \in \mathbf{X}^{(1,0)}$ as well as for $X, Y \in \mathbf{X}^{(0,1)}$

(IIIb) $R(X, \bar{Y}) Z=-h(X, Z) S(\bar{Y})$ for $X, Y, Z \in \mathbf{X}^{(1,0)}$ and its conjugate, namely,

$$
R(X, \bar{Y}) \bar{Z}=h(\bar{Y}, \bar{Z}) \bar{S}(X) \quad \text { for } X, Y, Z \in \mathbf{X}^{(1,0)} .
$$

(IVa) $\left(\nabla_{X} h\right)(Y, Z)=\left(\nabla_{Y} h\right)(X, Z)$ for $X, Y, Z \in \mathbf{X}^{(1,0)}$ and its conjugate, namely

$$
\left(\nabla_{X} h\right)(\bar{Y}, \bar{Z})=\left(\nabla_{\bar{Y}} h\right)(\bar{X}, \bar{Z}) .
$$

(IVb) $\left(\nabla_{\bar{Y}} h\right)(X, Z)=\tau(\bar{Y}) h(X, Z)$ for $X, Y, Z \in \mathbf{X}^{(0,1)}$ and its conjugate, namely

$$
\left(V_{X} h\right)(\bar{Y}, \bar{Z})=-\bar{\tau}(X) h(\bar{Y}, \bar{Z})
$$

for $X, Y, Z \in \mathbf{X}^{(1,0)}$.

Remark. (IIIa) reflects the same kind of property as the curvature tensor of a kählerian connection, namely, the equation (18), p. 157 in [K-N], vol II.

We shall have to derive some further basic equations.

We have from (IIb) and (IIc)

$$
\begin{gathered}
D_{X} D_{\bar{Y}} \zeta=D_{X}(-S(\bar{Y})+\tau(\bar{Y}) \zeta) \\
=-D_{X}(S(\bar{Y}))-h(X, S(\bar{Y})) \zeta+\left(\nabla_{X} \tau\right)(\bar{Y}) \zeta \\
D_{\bar{Y}} D_{X} \zeta=0
\end{gathered}
$$

and

$$
D_{[X, \bar{Y}]} \zeta=-S([X, \bar{Y}]) \zeta+\bar{\tau}([X, \bar{Y}]) .
$$

Since $[X, \bar{Y}]=\nabla_{X} \bar{Y}-\nabla_{\bar{Y}} X$, where $\nabla_{X} \bar{Y} \in \mathbf{X}^{(0,1)}$ and $\nabla_{\bar{Y}} X \in \mathbf{X}^{(1,0)}$, we have 


$$
S\left(\nabla_{X} \bar{Y}\right)=S([X, \bar{Y}]), \quad \tau\left(\nabla_{X} \bar{Y}\right)=\tau([X, \bar{Y}]) .
$$

We have therefore

$$
0=\left[D_{X}, D_{\bar{Y}}\right] \zeta-D_{[X, \bar{Y}]} \zeta=-\left(\nabla_{X} S\right)(\bar{Y})-h(X, S(\bar{Y})) \zeta+\left(\nabla_{X} \tau\right)(\bar{Y}) \zeta
$$

and hence for $X, Y \in \mathbf{X}^{(1,0)}$

$$
\left(\nabla_{X} S\right)(\bar{Y})=0
$$

and its conjugate

$$
\left(\nabla_{Y} \bar{S}\right)(X)=0
$$

We have also

$$
h(X, \bar{S}(\bar{Y}))=\left(\nabla_{X} \tau\right)(\bar{Y})
$$

and its conjugate

$$
h(\bar{S}(X), \bar{Y})=\left(\nabla_{\bar{Y}} \bar{\tau}\right)(\bar{X}) .
$$

The same kind of computation for $\left[D_{X}, D_{Y}\right] \bar{\zeta}-D_{[X, Y]} \bar{b}$ leads to

$$
\left(\nabla_{X} \bar{S}\right)(Y)-\bar{\tau}(X) \bar{S}(Y)=\left(\nabla_{Y} \bar{S}\right)(X)-\bar{\tau}(Y) \bar{S}(X)
$$

and its conjugate as well as (noting equation (Ib))

$$
\left(\nabla_{X} \bar{\tau}\right)(Y)-\left(\nabla_{Y} \bar{\tau}\right)(X)=0
$$

that is,

$$
(d \bar{\tau})(X, Y)=0
$$

for $X, Y \in \mathbf{X}^{(1,0)}$ and its conjugate.

We now consider the transversal (or normal) connection $\nabla^{\perp}$ and its curvature tensor $R^{\perp}$. First of all, from (IIa) we have $\nabla_{X}^{\perp} \zeta=0$ for $X \in \mathbf{X}^{(1,0)}$ and hence for $X, Y \in \mathbf{X}^{(1,0)}$

$$
R^{\perp}(X, Y) \zeta=0
$$

and its conjugate equation. From (IIb), (IIa) we have

$$
\begin{gathered}
\nabla_{\bar{Y}}^{\perp} \zeta=\tau(\bar{Y}) \zeta \\
D_{X}\left(\nabla_{\bar{Y}}^{\perp} \zeta\right)=X(\tau(\bar{Y}))=\left\{\left(\nabla_{X} \tau\right)(\bar{Y})+\tau\left(\nabla_{X} \bar{Y}\right)\right\} \zeta \\
\nabla_{X}^{\perp} \zeta=0, \quad \nabla_{\bar{Y}}^{\perp}\left(\nabla_{\bar{X}}^{\perp} \zeta\right)=0 \\
\nabla_{[X, \bar{Y}]}^{\perp} \zeta=\nabla_{\nabla_{X} \bar{Y}}^{\perp} \zeta=\left(\nabla_{X} \bar{Y}\right)
\end{gathered}
$$


again because $[X, \bar{Y}]=\nabla_{X} \bar{Y}-\nabla_{\bar{Y}} X$, where $\nabla_{\bar{Y}} X \in \mathbf{X}^{(1,0)}$ and $\nabla_{\bar{\sigma}_{\bar{Y}} X} \zeta=0$. From these we get $\left(X, Y \in \mathbf{X}^{(1,0)}\right)$

$$
R^{\perp}(X, \bar{Y}) \zeta=\left(\nabla_{X} \tau\right)(\bar{Y}) \zeta=2(d \tau)(X, \bar{Y}) \zeta .
$$

Here the last equality comes from the fact that

$$
\begin{aligned}
2 d \tau(X, \bar{Y}) & =X(\tau(\bar{Y}))-\bar{Y}(\tau(X))-\tau([X, \bar{Y}]) \\
& =X(\tau(\bar{Y}))-\tau\left(\nabla_{X} \bar{Y}-\nabla_{\bar{Y}} X\right) \\
& =X(\tau(\bar{Y}))-\tau\left(\nabla_{X} \bar{Y}\right)=\left(\nabla_{X} \tau\right)(\bar{Y})
\end{aligned}
$$

because $\tau(X)=0$ and $\tau\left(\nabla_{\vec{Y}} X\right)=0$. By taking the conjugate we get

$$
R^{\perp}(X, \bar{Y}) \bar{\zeta}=-\left(\nabla_{\bar{Y}} \bar{\tau}\right)(X) \bar{\zeta}=2(d \bar{\tau})(X, \bar{Y}) \bar{\zeta} .
$$

By a similar computation using equation (3.13) we get

$$
R^{\perp}(X, Y) \bar{\zeta}=0
$$

and its conjugate. We might also list

$$
d \tau(X, Y)=0
$$

corresponding to equation (3.14). Also the conjugate equation holds.

Summing up, we have

Codazzi equations and Ricci equations

(Va) $\left(\nabla_{X} S\right)(\bar{Y})=0$ for $X, Y \in \mathbf{X}^{(1,0)}$ and its conjugate.

(Vb) $\left(\nabla_{X} \bar{S}\right)(Y)-\bar{\tau}(X) \bar{S}(Y)=\left(\nabla_{Y} \bar{S}\right)(X)-\bar{\tau}(Y) \bar{S}(X) \quad$ for $\quad X, Y \in \mathbf{X}^{(1,0)}$ and its conjugate.

(Vc) $h(X, S(\bar{Y}))=\left(\nabla_{X} \tau\right)(\bar{Y})=2(d \tau)(X, \bar{Y}) \quad$ for $X, Y \in \mathbf{X}^{(1,0)}$ and its conjugate.

(VIa) $R^{\perp}(X, Y) \zeta=0$ for $X, Y \in \mathbf{X}^{(1,0)}$ and its conjugate. $d \tau(X, Y)=0$ for $X, Y \in \mathbf{X}^{(1,0)}$ and its conjugate.

(VIb) $R^{\perp}(X, Y) \bar{\zeta}=0$ for $X, Y \in \mathrm{X}^{(1,0)}$ and its conjugate. $d \bar{\tau}(X, Y)=0$ for $X, Y \in \mathbf{X}^{(1,0)}$ and its conjugate.

(VIc) $R^{\perp}(X, \bar{Y}) \zeta=2 d \tau(X, \bar{Y}) \zeta$ for $X, Y \in \mathbf{X}^{(1,0)}$ and its conjugate. $R^{\perp}(\bar{X}, Y) \bar{\zeta}=2(d \bar{\tau})(\bar{X}, \bar{Y}) \bar{\zeta}$.

Thus $d(X, \bar{Y})$ is a non-trivial term that appears in (Vc) and (VIc) in relation to $h$ and $R^{\perp}$.

For later use we now want to list these basic equations using the real representation; since the computation is quite similar to the previous one, we omit the detail. Starting from equation (1.1) and (1.5) and using 
the fact that for affine Kähler immersions $A J=-J A$ and $\nu=\mu \circ J$, we get the equations of Gauss, Codazzi and Ricci $(X, Y, Z$ are here real vector fields on $M)$ :

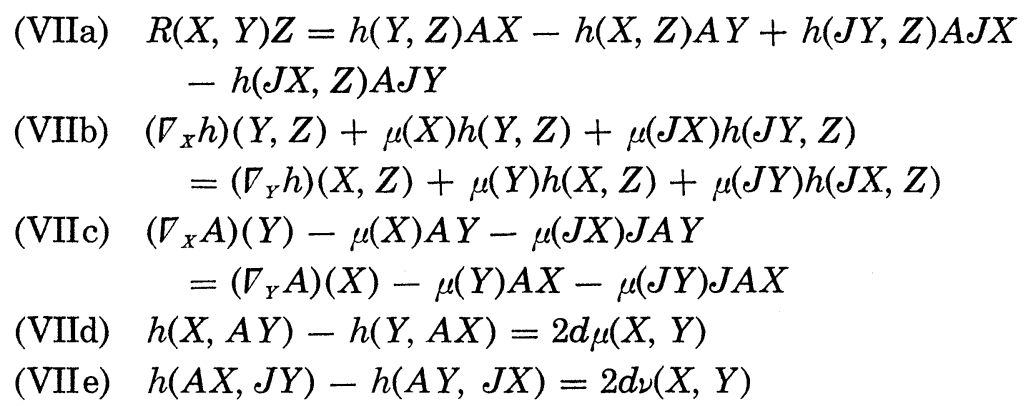

\section{§4. The fundamental theorem for affine Kähler immersions}

We now want to prove the fundamental theorem for affine Kähler immersions; throughout this section we shall use the real formalism, regarding the complex manifold $M^{n}$ as a $2 n$-dimensional real analytic manifold endowed with a complex structure $J$.

THEOREM 4.1. Let $M^{n}$ be an n-dimensional complex manifold with a complex structure $J$ together with an affine Kähler connection $\nabla$. Let $A$ be a real tensor field of type $(1,1)$ on $M^{n}$ together with a real symmetric tensor field $h$ of type $(0,2)$ and a 1-form $\mu$. We suppose that the following equations are satisfied $\left(X, Y, Z\right.$ are real vector fields on $\left.M^{n}\right)$ :

a) $A J=-J A$

b) $h(X, J Y)=h(J X, Y)$

c) $R(X, Y) Z=h(Y, Z) A X-h(X, Z) A Y+h(J Y, Z) A J X$ $-h(J X, Z) A J Y$

d) $\left(\nabla_{X} h\right)(Y, Z)+\mu(X) h(Y, Z)+\mu(J X) h(J Y, Z)$ $=\left(\nabla_{Y} h\right)(X, Z)+\mu(Y) h(X, Z)+\mu(J Y) h(J X, Z)$

e) $\left(\nabla_{X} A\right)(Y)-\mu(X) A Y-\mu(J X) J A Y$ $=\left(\nabla_{X} A\right)(X)-\mu(Y) A X-\mu(J Y) J A X$

f) $h(X, A Y)-h(Y, A X)=2 d \mu(X, Y)$

g) $h(A X, J Y)-h(A Y, J X)=2 d \nu(X, Y)$, with $\nu(X)=\mu(J X)$

where $R$ is the curvature tensor of $\nabla$. Then there exists locally a holomorphic immersion $f: M^{n} \rightarrow \mathbf{C}^{n+1}$ and an antiholomorphic (1,0)-transversal vector field $\zeta$ along $f$ so that $f:(M, \nabla) \rightarrow \mathbf{C}^{n+1}$ is an affine Kähler immersion. Such an immersion is unique up to a holomorphic affine transformation of 
$\mathbf{C}^{n+1}$. If $M^{n}$ is assumed to be simply connected, all the results above hold in a global setting.

Remark. We note that equations c), d), e), f), g) are precisely the Gauss, Codazzi and Ricci equations for an affine Kähler immersion according to formulas (VIIa, b, c, d, e) of $\S 3$, so that they are necessary for the existence of such an immersion.

Proof. We consider the trivial bundle $N=M^{n} \times \mathbf{R}^{2}$ over $M^{n}$, where $\mathbf{R}^{2}$ is endowed with the standard complex structure $J_{o}$. We define a con nection $\nabla^{\perp}$ on $N$ in the following way: we choose a nowhere vanishing section $\xi$ of $N$ and put for $X \in \mathbf{X}$

$$
\begin{gathered}
\nabla_{X}^{\perp} \xi=\mu(X) \xi+\mu(J X) J_{o} \xi \\
\nabla_{X}^{\perp} J_{o} \xi=J_{o} \nabla_{X}^{\perp} \xi .
\end{gathered}
$$

We can now define a connection $D^{\sharp}$ in the bundle $E=T M^{n} \oplus N$ as follows

$$
\begin{gathered}
D_{X}^{\sharp} Y=\nabla_{X} Y+h(X, Y) \xi-h(J X, Y) J_{o} \xi \\
D_{X}^{\sharp} \xi=-A X+\nabla_{\bar{X}} \xi \\
D_{X}^{*} J_{o} \xi=-J A X+J_{o} \nabla_{X}^{\frac{1}{X}} \xi .
\end{gathered}
$$

By using equations a)-f) it is easy to verify that the curvature operator of $D^{\sharp}$ vanishes identically; hence, by a standard result in the theory of connections in vector bundles (see [K-N], vol. I, Cor. 9.2, p. 92), we can find locally a $C^{\infty}$ bundle-isomorphism $\Phi$

$$
\Phi: E \rightarrow M^{n} \times \mathbf{R}^{(n+1)},
$$

which maps the connection $D^{\sharp}$ into the standard flat connection $D$ in the trivial bundle $M^{n} \times \mathbf{R}^{2(n+1)}$. We note that $I=J \oplus J_{0}$ is a complex structure along the fibres of $E$ and that $D^{\#}$ leaves $I$ parallel; since $\Phi$ is connection preserving, it follows that the complex structure $\tilde{J}=\Phi_{*} I$ along the fibres of $M^{n} \times \mathbf{R}^{2(n+1)}$ is parallel with respect to the connection $D$, so that we can speak of the complex structure $\tilde{J}$ induced on $\mathbf{R}^{2(n+1)}$ without ambiguity. In the following we shall identify $\left(\mathbf{R}^{2(n+1)}, \tilde{J}\right)$ with $\mathbf{C}^{n+1}$.

We denote by $\iota$ the inclusion $\iota: T M^{n} \hookrightarrow E$ and consider the map

$$
F=\Phi_{\circ}: T M^{n} \rightarrow M \times \mathbf{C}^{n+1} .
$$

If $X$ is any section of $T M^{n}$, i.e. any vector field on $M^{n}$, we can write 


$$
F\left(X_{x}\right)=\left(x, \theta_{x}(X)\right) \quad x \in M^{n}
$$

where $\theta$ is a $\mathbf{C}^{n+1}$-valued 1 -form on $M^{n}$. We first prove that $\theta$ is closed, i.e. $d \theta=0$. Indeed if $X$ and $Y$ are vector fields on $M^{n}$

$$
D_{X}^{*}(\iota Y)-D_{Y}^{*}(\iota X)=\iota([X, Y])
$$

since $\nabla$ is torsion-free and $h$ is symmetric. If we apply $\Phi$ to both members of (4.1) and use the fact that $\Phi$ is connection-preserving, we get

$$
X \theta(Y)-Y \theta(X)=\theta([X, Y])
$$

which means $d \theta=0$. Moreover we note that $\theta(J X)=i \theta(X)$ because $\Phi$ maps the complex structure $I$ into the complex structure of $\mathbf{C}^{n+1}$ given by the multiplication by $i$.

Therefore we can write $\theta$ as $\left(\theta^{1}, \cdots, \theta^{n+1}\right)$ where each $\theta^{i}$ is a $\mathbf{C}$-valued 1-form of type $(1,0)$ with $d \theta^{i}=0$; hence each $\theta^{i}$ may be expressed locally as $\theta^{i}=d f^{i}$ for some holomorphic function $f^{i}$ : indeed $\theta^{i}=\partial f^{i}+\overline{\hat{o}} f^{i}$ and from the fact that $\theta^{i}$ is of type $(1,0)$, we get that $\bar{\partial} f^{i}=0$, i.e. $f^{i}$ is holomorphic. We put $f=\left(f^{1}, \cdots, f^{n+1}\right)$ where $f: M^{n} \rightarrow \mathbf{C}^{n+1}$ is a holomorphic immersion: if $d f_{x}(X)=0$ for some $x \in M^{n}$ and $X \in T M_{x}^{n}$, then $F(X)=(x, 0)$ and $\iota(X)=\Phi^{-1}(0)=0$, hence $X=0$.

We need only to define the $(1,0)$-transversal vector field $\zeta$ by setting

$$
\zeta=\Phi(\xi)-i \tilde{J} \Phi(\xi)
$$

The condition of antiholomorphicity for $\zeta$ is actually equivalent to

$$
D_{X} \Phi(\xi)=\tilde{J} D_{J X} \Phi(\xi),
$$

and, since $\Phi$ is connection preserving, it is enough to check that

$$
D_{X}^{*} \xi=J D_{J}^{*} \xi
$$

But

$$
\begin{aligned}
J D_{J X}^{\sharp} \xi & =J\left(-A J X+\mu(J X) \xi-\mu(X) J_{o} \xi\right) \\
& =-J A J X+\mu(J X) J_{o} \xi+\mu(X) \xi \\
& =-A X+\mu(X) \xi+(J X) J_{o} \xi=D_{X}^{\sharp} \xi
\end{aligned}
$$

and this proves the first part of the theorem.

We now prove that such an immersion is unique up to a hlomorphic affine transformation of $\mathbf{C}^{(n+1)}$; the proof goes on as in the case of a kählerian hypersurface (see [K-N], vol II, p. 45). 
We suppose we have two (local) affine Kähler immersions $f, f^{\prime}: M^{n} \rightarrow$ $\mathbf{C}^{n+1}$ with antiholomorphic $(1,0)$-transversal vector fields $\zeta=\xi-i J \xi$ and $\zeta^{\prime}=\xi^{\prime}-i J \xi^{\prime}$ inducing on $M^{n}$ the same connection $\nabla$, shape operator $A$, fundamental form $h$ and normal connection form $\mu$. We fix a point $x_{o} \in M^{n}$ and choose a coordinate chart $U$, with real analytic coordinates $\left(x^{1}, \cdots, x^{2 n}\right)$, on which both $f$ and $f^{\prime}$ are defined. We put $e_{i}=f_{*}\left(\partial / \partial x^{i}\right)$ and $e_{i}^{\prime}=f_{*}^{\prime}\left(\partial / \partial x^{i}\right)(i=1, \cdots, 2 n)$ and define an affine transformation $B$ of $\mathbf{C}^{n+1}$ in the following way

$$
\begin{aligned}
& B\left(f\left(x_{o}\right)\right)=f^{\prime}\left(x_{o}\right) \\
& B\left(f\left(x_{o}\right)+e_{i}\left(x_{o}\right)\right)=f^{\prime}\left(x_{o}\right)+e_{i}^{\prime}\left(x_{o}\right) \\
& B\left(f\left(x_{o}\right)+\xi\right)=f^{\prime}\left(x_{o}\right)+\xi^{\prime} \\
& B\left(f\left(x_{o}\right)+J \xi\right)=f^{\prime}\left(x_{o}\right)+J \xi^{\prime} .
\end{aligned}
$$

Since $f$ and $f^{\prime}$ are holomorphic, $B$ turns out to be holomorphic. Hence by changing $f$ into $B \circ f$, we can suppose that $f\left(x_{o}\right)=f^{\prime}\left(x_{o}\right), e_{i}\left(x_{0}\right)=e_{i}^{\prime}\left(x_{0}\right)$ and $\xi\left(x_{o}\right)=\xi^{\prime}\left(x_{o}\right)$. If we denote by $\Gamma_{j k}^{i}$ the local Christoffel symbols of $\nabla$ and by $a_{i}^{k}$ the local matrix expression for $A$, we have that

$$
\begin{aligned}
& \frac{\partial e_{i}}{\partial x^{j}}=\Gamma_{i j}^{k} e_{k}+h\left(\partial / \partial x^{i}, \partial / \partial x^{j}\right) \xi-h\left(\partial / \partial x^{i}, J \partial / \partial x^{j}\right) J \xi \\
& \frac{\partial \xi}{\partial x^{i}}=-a_{i}^{k} e_{k}+\mu\left(\partial / \partial x^{i}\right) \xi+\mu\left(J \partial / \partial x^{i}\right) J \xi
\end{aligned}
$$

and the same for $\left(e_{i}^{\prime}, \cdots, e_{2 n}^{\prime}, \xi^{\prime}\right)$. Since $\left(e_{1}, \cdots, e_{2 n}, \xi\right)$ and $\left(e_{1}^{\prime}, \cdots, e_{2 n}^{\prime}, \xi^{\prime}\right)$ satisfy both the same system of set of differential equations and since their initial conditions coincide, the uniqueness of the solution for the Cauchy problem implies that $e_{i}=e_{i}^{\prime}$ on $U$, hence that $f=f^{\prime}$ on $U$.

If $M^{n}$ is assumed to be simply connected, then the mapping $\Phi$ is defined on the whole $E$ and also the function $f$ is globally defined on $M^{n}$; moreover if $f$ and $f^{\prime}$ are two such Kähler immersions, then by the previous argument we can find a holomorphic affine transformation of $\mathbf{C}^{n+1}$ so that $f \circ B$ and $f^{\prime}$ coincide locally, hence on the whole $M^{n}$ because of the holomorphicity.

q.e.d.

\section{§5. Flat normal connection and Ricci-flatness}

Unlike the classical theory for non-degenerate hypersurfaces in $\mathbf{R}^{n+1}$ (see [N-Pi]-1), it is not generally possible to choose for a complex hypersurface in $\mathbf{C}^{n+1}$ an antiholomorphic $(1,0)$-transversal vector field such that 
$\tau$ and $\bar{\tau}$ are identically zero. In the Kähler case, it is known that a complex hypersurface $M^{n}$ in a Kähler manifold $M^{n+1}$ of constant sectional curvature $c$ has flat normal connection only if $c=0$ and $M^{n}$ is totally geodesic (see Theorem 7 in [N-S]). In the affine Kähler case we have

Proposition. Let $M^{n}$ be a complex hypersurface in $\mathbf{C}^{n+1}$ and $\zeta$ an antiholomorphic $(1,0)$-transversal vector field.

1) The Ricci tensor of $M^{n}$ vanishes identically if and only if the normal connection is flat, i.e. $R^{\perp}=0$.

2) Suppose the normal connection is flat. Then we may find locally an antiholomorphic function $\phi$ such that for the antiholomorphic transversal vector field $\phi \zeta$, the normal connection form vanishes identically.

Proof. Note that the Ricci tensor is defined for $Y, Z \in \mathbf{X}^{c}$ by

$$
\operatorname{Ric}(Y, Z)=\operatorname{tr}\left\{X \in T^{c} \mapsto R(X, Y) Z \in \mathbf{X}^{c}\right\} .
$$

For an affine Kähler connection, we know that $R(X, Y)=0$ for $X, Y \in \mathbf{X}^{(1,0)}$; this implies that $\operatorname{Ric}(Y, Z)=\operatorname{Ric}(\bar{Y}, \bar{Z})=0$ for $X, Y \in \mathbf{X}^{(1,0)}$. So for $Y, Z$ $\in \mathbf{X}^{(1,0)}$ we have

$$
\operatorname{Ric}(\bar{Y}, Z)=\operatorname{tr}\left\{X \in \mathbf{X}^{(1,0)} \mapsto R(X, \bar{Y}) Z \in \mathbf{X}^{(1,0)}\right\},
$$

which is equal to $-h(S(\bar{Y}), Z)$ by equation (IIIb). By equation (Vc), we see that $\operatorname{Ric}(\bar{Y}, Z)=0$ if and only if $d \tau(\bar{Y}, Z)=0$. This proves 1$)$ by equations (VIa)-(VIc).

To prove 2), we first remark that if $\phi$ is an antiholomorphic function, then $\zeta_{1}=\phi \zeta$ is antiholomorphic. The normal connection form $\tau_{1}$ can be found as follows. On the one hand, we have

$$
D_{Z} \zeta_{1}=\left(\bar{Z}_{\phi}\right) \zeta+\phi\left(D_{\bar{Z}} \zeta\right)=\left(\bar{Z}_{\phi}+\phi \tau(\bar{Z})\right) \zeta-\phi f_{*}(S(\bar{Z})) .
$$

On the other hand

$$
D_{Z} \zeta_{1}=-f_{*}\left(S_{1}(\bar{Z})\right)+\tau_{1}(\bar{Z}) \zeta_{1}=-f_{*}\left(S_{1}(\bar{Z})\right)+\phi \tau_{1}(\bar{Z}) .
$$

Hence we get

$$
\tau_{1}(\bar{Z})=\tau(\bar{Z})+(\bar{Z} \phi) / \phi .
$$

for every $Z \in \mathbf{X}^{(1,0)}$. Now we start with the assumption that $R^{\perp}=0$. By the equations (VIa, b, c) we see that $d \tau=0$. Since $\tau$ is a 1 -form of type $(0,1)$ that is zero on $(1,0)$-vectors, we can locally write $\tau=\sum_{k=1}^{n} \psi^{k} d \bar{z}^{k}$ hence 


$$
d \tau=\sum_{m, k=1}^{n}\left(\partial \psi^{k} / \partial z^{m}\right) d z^{m} \wedge d \bar{z}^{k}+\sum_{k, m=1}^{n}\left(\partial \psi^{k} / \partial \bar{z}^{m}\right) d \bar{z}^{n} \wedge d \bar{z}^{k}=0 .
$$

Evaluating $d \tau$ on $\left(\partial / \partial z^{m}, \partial / \partial \bar{z}^{k}\right)$ as well as on $\left(\partial / \partial \bar{z}^{m}, \partial / \partial \bar{z}^{k}\right)$, we get that $\partial \psi^{k} / \partial z^{m}=0$ and $\partial \psi^{k} / \partial \bar{z}^{m}=0$ so that each $\psi^{k}$ is a constant function, say, equal to $a^{k}$. We have that $\tau=\sum_{k=1}^{n} a^{k} d \bar{z}^{k}=d \bar{\psi}$, where $\psi$ is the holomorphic function $\psi=\sum_{k=1}^{n} \bar{a}^{k} z^{k}$. Let $\phi=\exp (-\bar{\psi})$ which is an antiholomorphic function. Then we have

$$
\tau\left(\partial / \partial \bar{z}^{k}\right)+\left(\partial \phi / \partial \bar{z}^{k}\right) / \phi=a^{k}-\partial \bar{\psi} / \partial z^{k}=a^{k}-a^{k}=0 .
$$

By (5.1) this means that $\tau_{1}=0$ and this proves 2).

q.e.d.

We now prove

Theorem 5.1. Let $M^{n}$ be a complex hypersurface in $\mathbf{C}^{n+1}$ with an antiholomorphic $(1,0)$-transversal vector field $\zeta$. Suppose the second fundamental form $h$ is non-degenerate (which condition is independent of the choice of any transversal $(1,0)$-vector field, holomorphic or antiholomorphic or whatever). Then the following conditions are equivalent to each other:

1) The Ricci tensor of $M^{n}$ vanishies identically;

2) the normal connection of $M^{n}$ is flat;

3) $\zeta$ is parallel in $\mathbf{C}^{n+1}$ and thus $M^{n}$ is equivalent to the graph of a certain holomorphic function $F\left(z^{1}, \cdots, z^{n}\right)$ over a domain $D$ in $\mathbf{C}^{n}$ with non-degenerate Hessian.

Proof. The equivalence of 1) and 2) has been already proved. Let us assume 2). By the previous proposition we may rechoose $\zeta$ so that $\tau$ is identically zero. We also know that $h(S(\bar{Y}), Z)=0$ for all $Y, Z \in \mathbf{X}^{(1,0)}$. Since $h$ is non-degenerate, this means that $S=0$. Thus

$$
D_{Z} \zeta=-f_{*}(S(\bar{Z}))+\tau(\bar{Z}) \zeta=0,
$$

that is, $\zeta$ is parallel in $\mathbf{C}^{n+1}$. It follows that $M^{n}$ is the graph of a certain holomorphic function $F$ on a domain $D$ in a hyperplane transversal to the constant vector $\zeta$ in $\mathbf{C}^{n+1}$. In this case, $h$ on $\mathbf{X}^{(1,0)} \times \mathbf{X}^{(1,0)}$ is expressed by the Hessian matrix of $F$, which is non-degenerate. Thus 2) implies 3). The converse, that is, 3) implies 1), follows from the fact that for a graph immersion the shape operator $S$ vanishes identically. q.e.d.

If $M^{n}$ is assumed to be flat, that is, $R=0$, then of course the Ricci tensor is zero and we get $M^{n}$ to be a graph. However we can prove this result without assuming that $h$ is non-degenerate. 
TheOREM 5.2. Let $f:\left(M^{n}, \nabla\right) \rightarrow \mathbf{C}^{n+1}$ be an affine Kähler immersion. If $M^{n}$ is flat, that is, $R=0$, then $f$ is totally geodesic or a graph immersion with parallel $(1,0)$-transversal vector field.

Proof. By equation (IIIb), $R=0$ implies that $h(Y, Z) S(Y)=0$ for all $X, Y, Z \in \mathbf{X}^{(1,0)}$. Suppose $S \neq 0$ at a point $x \in M^{n}$. Then by picking $Y \in \mathbf{X}^{(1,0)}$ with $S(\bar{Y}) \neq 0$, we find that $h$ vanishes identically on $\mathbf{X}^{(1,0)} \times \mathbf{X}^{(1,0)}$ at $x$, hence on $\mathrm{X}^{\mathrm{C}} \times \mathrm{X}^{\mathrm{C}}$ at $x$. Suppose $S$ is not identically zero. Then $S \neq 0$ at a point $x$ and hence in a neighborhood $U$ of $x$. By the above argument, we see that $h=0$ on $U$. But $h$ is real analytic and therefore it vanishes on $M^{n}$. This means that $M^{n}$ is totally geodesic. On the other hand, if $S$ is identically zero, then $f$ is a graph immersion because we already know that, due to $R=0$ we may rechoose an antiholomorphic $(1,0)$ transversal vector field with $\tau=\bar{\tau}=0$.

q.e.d.

\section{§6. An analogue of Berwald's theorem}

Let $f:(M, \nabla) \rightarrow \mathbf{C}^{n+1}$ be an affine Kähler immersion. Among the covariant derivatives of $h$ the non trivial ones are of the form $\left(\nabla_{X} h\right)(Y, Z)$, $\left(\nabla_{\bar{X}} h\right)(Y, Z)$ for $X, Y, Z \in \mathbf{X}^{(1,0)}$ and their conjugate. For these we have equations (IVa) and (IVb).

We prove an analogue of Berwald's Theorem (see [N-P]-2)

THEOREm 6.1. Let $f:(M, \nabla) \rightarrow \mathbf{C}^{n+1}$ be a non-degenerate affine Kähler immersion. If $\left(\nabla_{X} h\right)(Y, Z)$ are zero for all complex vector fields $X, Y, Z$, then $f$ is equivalent to the graph of a quadratic polynomial

$$
F\left(z^{1}, \cdots, z^{n}\right)=\sum_{j, k=1}^{n} a_{j k} z^{j} z^{k}
$$

where $\operatorname{det}\left[a_{j k}\right] \neq 0$.

Proof. From equation (VIb), we obtain $\tau(\bar{Y}) h(X, Y)=0$ for all $X, Y$, $Z \in \mathbf{X}^{(1,0)}$. Thus we get $\tau=0$. From equation (VIa) we get that $h(X, S(\bar{Z})$ ) $=0$ and since $h$ is non-degenerate, we conclude that $S=0$. From the fact that $S$ and $\tau$ vanish identically, we may use the previous argument to choose an antiholomorphic (1,0)-transversal vector field which is parallel in $\mathbf{C}^{n+1}$. We see that $f$ is a graph immersion

$$
f:\left(z^{1}, \cdots, z^{n}\right) \in D \mapsto\left(z^{1}, \cdots, z^{n}, F\left(z^{1}, \cdots, z^{n}\right)\right)
$$

where $F$ is a holomorphic function defined on a domain $D$ in $\mathbf{C}^{n}$ and 
$\zeta=(0, \cdots, 0,1)$. From this we have

$$
h\left(\partial / \partial z^{j}, \partial / \partial z^{k}\right)=F_{j k}\left(=\partial^{2} F / \partial z^{j} \partial z^{k}\right)
$$

and

$$
\left(\nabla_{\partial / \partial z^{m}} h\right)\left(\partial / \partial z^{j}, \partial / \partial z^{k}\right)=\partial F_{j k} / \partial z^{m}=0 .
$$

Hence each $F_{j k}$ is a constant and

$$
F=\frac{1}{2} \sum_{j k=1}^{n} a_{j k} z^{j} z^{k}+\sum_{m=1}^{n} b_{m} z^{m}+c
$$

for some constants $b_{m}$ and $c$.

q.e.d.

\title{
REFERENCES
}

[A] K. Abe, Affine geometry for complex hypersurfaces, to appear.

[D-V-V] F. Dillen, L. Vrancken and L. Verstraelen, Complex affine differential geometry, Atti. Accad. Peloritana Pericolanti Cl. Sci. Fis. Mat. Nat. LXVI (1988), 231-260.

[K-N] S. Kobayashi and K. Nomizu, Foundations of Differential Geometry, vol I, II John John Wiley, New York, 1963, 1969.

[N-Pi]-1 K. Nomizu and U. Pinkall, On the geometry of affine immersions, Math. Z., 195 (1987), 165-178.

[N-Pi]-2 - Cubic form theorem for affine immersions, Results in Math., 13 (1988), 338-362.

[N-Po] K. Nomizu and F. Podestà On affine Kähler structures, Bull. Soc. Math. Belg. 41 (1989) Ser. B, 275-281.

[N-S] K. Nomizu and B. Smyth, Differential geometry for complex hypersurfaces II, J. Math. Soc. Japan, 20 (1968), 498-521.

\author{
Katsumi Nomizu \\ Department of Mathematics \\ Brown University \\ Providence, RI 02912 \\ U.S.A. \\ Ulrich Pinkall \\ Technische Universität Berlin \\ Strasse des 17. Juni 136 \\ D-1000 Berlin 12 \\ F.R.G. \\ Fabio Podestà \\ c/o Scuola Normale Superiore \\ Piazza Cavalieri 7 \\ I-56100 Pisa \\ Italy
}

